\title{
O fenômeno do suicídio indígena no Brasil: revisão integrativa da literatura
}

\author{
The phenomenon of indigenous suicide in Brazil: integrative literature review \\ El fenómeno del suicidio indígena en Brasil: revisión integrativa de la literatura
}

Anderson Gomes de Oliveira ${ }^{1}$, Carla Andreele Moraes Lima da Silva ${ }^{1}$, Ilrislanny Montenegro Costa Silva $^{1}$, Adriana Duarte de Sousa ${ }^{2 *}$, Neila França Leite $^{2}$, Altair Seabra de Farias ${ }^{2}$, Ana Beatriz Souza e Souza ${ }^{1}$, Francisco Mailon Pereira Barroso ${ }^{2}$, Kátia Cilene Rodrigues Ferreira ${ }^{1}$, Maria de Lourdes da Cruz Braga'.

\section{RESUMO}

Objetivo: Descrever o fenômeno do suicídio indígena no Brasil. Métodos: Trata-se de revisão integrativa da literatura, que buscou descrever o fenômeno do suicídio de populações indígenas no Brasil, a partir de publicações científicas indexadas nas bases de dados Scientific Electronic Library Online (SCIELO); Literatura Latino-Americana em Ciências da Saúde (LILACS) e Literatura Internacional em Ciências da Saúde (MEDLINE) entre janeiro de 2010 a dezembro de 2018, cuja amostra final resultou em 6 artigos. Resultados: O perfil do indígena suicida é similar em todos os contextos estudados. A morte frequentemente ocorre nos domicílios e o método preferencial é o enforcamento seguido por envenenamento. Elementos da cosmologia indígena como o "enfeitiçamento", "estrago" e "inveja" que, teriam como objetivo causar malefícios à pessoa a qual eles foram destinados, também figuram como causadores do suicídio. Por fim, a própria forma como é representado o post-mortem do suicida, apontam para as especificidades encontradas na significação do auto-extermínio neste contexto. Considerações finais: O suicídio entre indígenas configura-se como um grave problema de saúde pública, pois além das questões já conhecidas, necessitamos levar em consideração as caraterísticas locorregionais, de populações etnicamente diferenciadas.

Palavras-chave: Suicídio, Povos indígenas, Saúde indígena.

\begin{abstract}
Objective: To describe the phenomenon of indigenous suicide in Brazil. Methods: This is an integrative literature review, which sought to describe the phenomenon of suicide among indigenous populations in Brazil, based on scientific publications indexed in the Scientific Electronic Library Online (SCIELO) databases; Latin American Literature in Health Sciences (LILACS) and International Literature in Health Sciences (MEDLINE) between January 2010 and December 2018, whose final sample resulted in 6 articles. Results: The profile of the suicidal indigenous is similar in all contexts. Death often occurs in households and the preferred method is hanging followed by poisoning. Elements of indigenous cosmology such as "bewitching", "damage" and "envy" that would aim to cause harm to the person to whom they were destined, also appear as causing suicide. Finally, the very way in which the suicide's post-mortem is represented, point to the specificities found in the meaning of self-extermination in this context. Final considerations: Suicide among indigenous people is a serious public health problem, because in addition to the issues already known, we need to take into account the local and regional characteristics of ethnically different populations.
\end{abstract}

Keywords: Suicide, Indigenous people, Indigenous health.

\section{RESUMEN}

Objetivo: describir el fenómeno del suicidio indígena en Brasil. Métodos: Se trata de una revisión integrativa de la literatura, que buscó describir el fenómeno del suicidio entre las poblaciones indígenas en Brasil, basado en publicaciones científicas indexadas en las bases de datos de la Biblioteca Electrónica Científica en línea

1 Centro Universitário do Norte (UNINORTE), Manaus - AM

2 Universidade do Estado do Amazonas (UEA), Manaus - AM. *E-mail: adrianaduartedesousa@hotmail.com

SUBMETIDO EM: $6 / 2020 \quad$ ACEITO EM: 7/2020 | PUBLICADO EM: 9/2020 
(SCIELO); Literatura Latinoamericana en Ciencias de la Salud (LILACS) y Literatura Internacional en Ciencias de la Salud (MEDLINE) entre enero de 2010 y diciembre de 2018, cuya muestra final resultó en 6 artículos. Resultados: El perfil de los indígenas suicidas es similar en todos los contextos estudiados. La muerte a menudo ocurre en los hogares y el método preferido es colgar seguido de envenenamiento. Elementos de la cosmología indígena como "hechizar", "dañar" y "envidiar" que pretenden causar daño a la persona a la que están destinados, también parecen causar suicidio. Finalmente, la forma misma en que se representa la autopsia del suicidio, señala las especificidades encontradas en el significado de autoexterminación en este contexto. Consideraciones finales: El suicidio entre los pueblos indígenas es un grave problema de salud pública, porque además de los problemas ya conocidos, debemos tener en cuenta las características locorregionales de poblaciones étnicamente diferentes.

Palabras clave: Suicidio, Pueblos indígenas, Salud indígena.

\section{INTRODUÇÃO}

Segundo a Organização Mundial de Saúde (OMS) e a Organização Pan Americana da Saúde (OPAS), aproximadamente um milhão de pessoas cometem suicídio a cada ano, o que equivale a uma morte a cada 40 segundos, sendo que a cada três segundos uma pessoa atenta contra a própria vida (OPAS, 2018; OMS, 2014). Em muitos países, o suicídio é uma das três principais causas de mortes de adolescentes e jovens com idade entre 15 e 29 anos. Mundialmente, a taxa de mortalidade por suicídio aumentou em $60 \%$ na última metade do século XX (BOTEGA NJ, 2009).

O Brasil, desde a década de 1960, passa uma transição epidemiológica, onde os óbitos por causas externas aumentaram consideravelmente em relação às doenças infecciosas e parasitárias. O crescimento nas taxas de suicídio tem colaborado com essa tendência, sendo essa a terceira causa de óbito por fatores externos identificados: homicídio $(36,4 \%)$, óbitos relacionados ao trânsito $(29,3 \%)$ e suicídio $(6,8 \%)$ (REICHENHEIM ME, et al., 2011).

Segundo o Boletim Epidemiológico do Ministério da Saúde, até final de 2017 o Brasil encontrava-se em oitavo lugar, dentre os países com maior número de casos de suicídio registrados. No entanto, quando analisamos os números de suicídio no país, estabelecemos uma conexão importante se compararmos as regiões do Brasil com maior número de mortes por suicídio (Centro-Oeste, Sul e Norte) (BRASIL, 2017).

Apesar das regiões citadas acima apresentarem importantes diferenças concernentes aos aspectos econômicos, demográficos e culturais, deve-se destacar que existem evidências de que o suicídio no Amazonas e Mato Grosso do Sul, estaria intimamente relacionado à presença de populações indígenas (WAISELFISZ JJ, 2011).

Souza MLP e Orellana JDY (2012) analisaram a taxa de mortalidade por suicídio entre populações indígenas e não indígenas nas cinco macrorregiões brasileiras, do período de 2006 a 2010 e concluíram que o suicídio é um grande problema de saúde no Brasil, especialmente entre os povos indígenas. Três municípios destacaram-se, pois apresentaram números considerados preocupantes: Santa Isabel do Rio Negro, com taxa bruta de suicídio de com 36,4/100,000 habitantes; São Gabriel da Cachoeira, com taxa bruta de 27,6/100,000 habitantes e; Tabatinga, com taxa bruta de 25,2/100,000 habitantes (SOUZA MPL e ORELLANA JDY, 2012).

Ainda que os municípios citados sejam considerados de pequeno porte e o somatório de seus contingentes populacionais represente apenas 3,1\% do total do Estado do Amazonas, neles foram registrados $18,4 \%$ dos suicídios ocorridos no Estado, evidenciando não só a importância do problema nas regiões do Alto Rio Solimões (onde está localizado o município de Tabatinga) e Alto Rio Negro (onde estão localizados os municípios de Santa Isabel do Rio Negro e São Gabriel da Cachoeira) (SOUZA MPL e ORELLANA JDY, 2013).

Observa-se que o suicídio é considerado um problema de saúde pública em vários países do mundo, ocorrendo inclusive entre povos culturalmente diferenciados e, por ser uma temática pouco explorada, 
encontrava-se oculta e ignorada. Desta forma, este trabalho teve como objetivo descrever através de uma revisão integrativa de literatura o fenômeno do suicídio de populações indígenas no Brasil.

\section{MÉTODOS}

O presente estudo se caracteriza como uma revisão integrativa da literatura que buscou apresentar a revisão e análise crítica acerca da temática do suicídio indígena no Brasil. Esse método proporciona a síntese de conhecimento e a incorporação da aplicabilidade de resultados de estudos significativos na prática (SOUZA MT, et al., 2010).

Para tanto, foram adotadas as seis etapas indicadas para a constituição da revisão integrativa da literatura: 1) identificação do tema e seleção da hipótese ou questão de pesquisa para a elaboração da revisão integrativa; 2) estabelecimento de critérios para inclusão e exclusão de estudos/ amostragem ou busca na literatura; 3) definição das informações a serem extraídas dos estudos selecionados/categorização dos estudos; 4) avaliação dos estudos incluídos na revisão integrativa; 5) interpretação dos resultados e; 6) apresentação da revisão/síntese do conhecimento (GANONG LH, 1987).

A busca foi realizada entre os meses de julho e agosto de 2019, utilizando-se os bancos de dados da LILACS (Literatura LatinoAmericana e do Caribe em Ciências da Saúde), MEDLINE (Literatura Internacional em Ciência da Saúde) e SCIELO (Scientific Electronic Library Online). Para realizar as buscas foram utilizados os seguintes descritores: "Suicídio", "Povos Indígenas", "prevalência". Todos disponíveis nos Descritores em Ciências da Saúde (DeCS).

Os critérios de inclusão estabelecidos foram: artigos originais disponibilizados na íntegra e na forma online, publicados no idioma português no período compreendido entre os anos de 2010 e 2018. Como critérios de exclusão foi estabelecido artigos de pesquisa bibliográfica e de reflexão, teses, dissertações e artigos repetidos em diferentes bases de dados.

Do material obtido, 46 artigos, procedeu-se à leitura minuciosa de cada artigo, destacando aqueles que responderam ao objetivo proposto por este estudo, a fim de organizar e tabular os dados. Para a organização e tabulação dos dados, os autores elaboraram instrumento de coleta de dados contendo: título, periódico, ano de publicação, categoria do estudo, natureza do estudo, referencial teórico, método de análise e principais resultados.

Seguindo os critérios de inclusão, 6 estudos foram selecionados para compor a amostra final. Os trabalhos foram comparados e agrupados por similaridade de conteúdo, sob a forma de categorias empíricas, sendo construídas quatro categorias para análise, assim especificadas: Suicídio indígena: caracterizando o fenômeno, Suicídio indígena: métodos utilizados, Suicídio indígena e o pós-morte: o que acontece com quem se mata? e Suicídio indígena: uma problemática de saúde pública oculta.

\section{RESULTADOS}

Inicialmente foram coletados 46 artigos nas bases de dados selecionadas e após aplicação dos critérios de inclusão e exclusão, a amostra final totalizou 6 artigos. Os artigos selecionados têm como interesse explanar/analisar sobre o fenômeno do suicídio entre as populações indígenas no país.

Grande parte dos artigos selecionados são provenientes de estudos descritivos e essencialmente quantitativos. No entanto, destacamos a importância/necessidade em nos debruçarmos sobre as pesquisas com abordagem qualitativa, como forma de compreender o tema em profundidade.

As informações relevantes das publicações selecionadas foram extraídas e sumarizadas em um quadro denominado "Identificação e descrição do conteúdo do artigo" (FONSECA RMP, 2008). Os resultados serão apresentados e discutidos na seguinte ordem: base, revista, título, autor(es), objetivo, metodologia e ano de publicação, conforme evidenciando no Quadro 1. 
Quadro 1 - Identificação e descrição do conteúdo do artigo.

\begin{tabular}{|c|c|c|c|c|c|c|c|c|}
\hline \multirow[b]{2}{*}{ № } & \multirow[b]{2}{*}{ Base } & \multirow[b]{2}{*}{ Revista } & \multirow[b]{2}{*}{ Título } & \multirow[b]{2}{*}{ Autor (es) } & \multirow[b]{2}{*}{ Objetivo } & \multicolumn{2}{|c|}{ Metodologia } & \multirow[b]{2}{*}{ Ano } \\
\hline & & & & & & $\begin{array}{l}\text { Tipo de } \\
\text { estudo }\end{array}$ & Abordagem & \\
\hline 1 & LILACS & $\begin{array}{l}\text { Revista } \\
\text { Saúde e } \\
\text { Sociedade }\end{array}$ & $\begin{array}{c}\text { Narrativas indígenas sobre } \\
\text { suicídio no Alto Rio Negro, Brasil: } \\
\text { tecendo sentidos. }\end{array}$ & SOUZA MLP. & $\begin{array}{l}\text { Analisar sete narrativas sobre o } \\
\text { suicídio de um Kumu (curandeiro } \\
\text { tradicional) da mais populosa } \\
\text { comunidade indígena de São Gabriel } \\
\text { da Cachoeira. }\end{array}$ & $\begin{array}{l}\text { Transversal } \\
\text { Analítico- } \\
\text { interpretativo }\end{array}$ & Qualitativo & 2016 \\
\hline 2 & LILACS & $\begin{array}{l}\text { Revista } \\
\text { Saúde e } \\
\text { Sociedade }\end{array}$ & $\begin{array}{l}\text { Jurupari se suicidou? notas para } \\
\text { investigação do suicídio no } \\
\text { contexto indígena. }\end{array}$ & $\begin{array}{l}\text { SOUZA MLP } \\
\text { e FERREIRA } \\
\text { LO }\end{array}$ & $\begin{array}{l}\text { Refletir sobre as dificuldades para } \\
\text { utilização do conceito de suicídio no } \\
\text { contexto indígena, ponto crucial para } \\
\text { abordagem deste tema através de } \\
\text { uma perspectiva menos } \\
\text { etnocêntrica. }\end{array}$ & $\begin{array}{l}\text { Transversal } \\
\text { Analítico- } \\
\text { interpretativo }\end{array}$ & Qualitativo & 2014 \\
\hline 3 & LILACS & $\begin{array}{l}\text { Jornal } \\
\text { Brasileiro de } \\
\text { Psiquiatria }\end{array}$ & $\begin{array}{c}\text { Desigualdade na mortalidade por } \\
\text { suicídio entre indígenas e não } \\
\text { indígenas no estado do } \\
\text { Amazonas, Brasil. }\end{array}$ & $\begin{array}{l}\text { SOUZA MLP } \\
\text {,et al. }\end{array}$ & $\begin{array}{l}\text { Analisar as taxas e algumas } \\
\text { características da mortalidade por } \\
\text { suicídio entre indígenas e não } \\
\text { indígenas no Amazonas. }\end{array}$ & $\begin{array}{l}\text { Transversal } \\
\text { Analítico- } \\
\text { descritivo }\end{array}$ & Quantitativo & 2013 \\
\hline 4 & LILACS & $\begin{array}{l}\text { Revista } \\
\text { Psicologia } \\
\text { Ciência e } \\
\text { Profissão }\end{array}$ & $\begin{array}{c}\text { Suicídios de jovens } \\
\text { Guarani/Kaiowá de Mato Grosso } \\
\text { do Sul, Brasil. }\end{array}$ & $\begin{array}{l}\text { GRUBITS S, } \\
\text { et al. }\end{array}$ & $\begin{array}{l}\text { Apresentar conclusões de estudos } \\
\text { referentes às causas do suicídio } \\
\text { entre os jovens Guarani/Kaiowá. }\end{array}$ & $\begin{array}{l}\text { Transversal } \\
\text { Analítico- } \\
\text { descritivo }\end{array}$ & Quantitativo & 2011 \\
\hline 5 & SCIELO & $\begin{array}{l}\text { Revista } \\
\text { Epidemiologia } \\
\text { e Serviço de } \\
\text { Saúde }\end{array}$ & $\begin{array}{l}\text { Caracterização da mortalidade por } \\
\text { suicídio entre indígenas e não } \\
\text { indígenas em Roraima, Brasil, } \\
2009-2013\end{array}$ & $\begin{array}{l}\text { SOUZA MLPJ } \\
\text { e RICARDO } \\
\text { TSO. }\end{array}$ & $\begin{array}{l}\text { Descrever características e taxas da } \\
\text { mortalidade por suicídio entre } \\
\text { indígenas e não indígenas em } \\
\text { Roraima, Brasil. }\end{array}$ & $\begin{array}{l}\text { Transversal } \\
\text { Analítico- } \\
\text { descritivo }\end{array}$ & Quantitativo & 2017 \\
\hline 6 & SCIELO & $\begin{array}{l}\text { Revista } \\
\text { Brasileira de } \\
\text { Epidemiologia }\end{array}$ & $\begin{array}{c}\text { Mortalidade por Suicídio: um } \\
\text { enfoque em municípios com alta } \\
\text { proporção de população } \\
\text { autodeclarada indígena no Estado } \\
\text { do Amazonas, Brasil. }\end{array}$ & $\begin{array}{l}\text { ORELLANA, } \\
\text { JDY, et al. }\end{array}$ & $\begin{array}{l}\text { Analisar as taxas de mortalidade e } \\
\text { descrever as características } \\
\text { demográficas e epidemiológicas dos } \\
\text { suicídios registrados no Estado do } \\
\text { Amazonas }\end{array}$ & $\begin{array}{l}\text { Transversal } \\
\text { Analítico- } \\
\text { descritivo }\end{array}$ & Quantitativo & 2013 \\
\hline
\end{tabular}

Fonte: OLIVEIRA AG, et al., 2020. do Amazonas, Brasil. 


\section{DISCUSSÃO}

Após a análise dos resultados a discussão foi organizada em categorias empíricas. Cada categoria nos possibilitou produzir um recorte analítico que favoreceu a compreensão de aspectos históricos, fatores de risco ou protetores, fatores agravantes (doença mental prévia), circunstâncias afetivas, sociais e econômicas, vida pessoal e social e possíveis motivações para o suicídio indígena.

\section{Suicídio indígena: caracterizando o fenômeno}

Em relação a caracterização do suicídio em populações indígenas, os textos selecionados demonstraram que o mesmo se caracteriza como um fenômeno majoritariamente juvenil, com faixa etária que varia de 10 a 19 anos.

Tais dados coadunam com estudos realizados na Região do Alto Rio Negro-AM, como uma pesquisa realizada por Souza MPL e Orellana JDY (2012), que teve por objetivo descrever as características e as taxas brutas de mortalidade por suicídio em São Gabriel da Cachoeira e que demonstrou que as taxas mais elevadas foram observadas no sexo masculino e nas faixas etárias de 15-24 e 25-34 anos.

O relatório intitulado "Suicídios indígenas no município de São Gabriel da Cachoeira (2001/2011)", elaborado a pedido do Ministério Público do Amazonas - MP/AM, como forma de esclarecer as ocorrências cada vez maiores de casos de suicídios entre jovens e adolescentes no município de São Gabriel da Cachoeira também contém informações semelhantes àquelas encontradas na amostra da presente pesquisa (COUTINHO W, 2011).

Grubits S, et al. (2011) em estudo realizado junto aos Guarani/Kaiowá em Mato Grosso do Sul, mostrou que a maioria dos suicidas é de homens, apesar do percentual de mulheres estar próximo. Os suicídios ocorreram com mais frequência nas faixas etárias abaixo de 29 anos.

Erthal RMC (2001), também encontrou predominância do sexo masculino entre os casos de suicídio registrados entre os Tikuna do Alto Solimões, com idades que variavam de 16 a 25 anos.

A autora elencou as diferentes faixas etárias dos homens Tikuna, apontando as responsabilidades, deveres e qualidades esperadas ou assumidas por cada um deles no decorrer de seus ciclos de vida. Questões como a continuidade ou não da vida escolar, busca por trabalho remunerado dentro e fora das comunidades indígenas, alistamento no serviço militar, firmação do compromisso com as estruturas políticas e religiosas do clã do qual fazem parte, casamento, trabalho tanto na roça ou na pesca de subsistência, podem ou não influenciar os casos de suicídio registrados nesta etnia (ERTHAL RMC, 2001).

O estudo de representações sociais realizado por Pereira MM (2013), na região do Alto Rio Negro, apontou o mesmo perfil de indivíduos (jovens e adolescentes do sexo masculino) como possuidor de alguma característica que "fragilizaria" ou os tornaria mais "vulneráveis" que outros grupos.

Segundo Erthal RMC (2001), essa fase parece ser a época da vida onde o jovem enfrenta diversas situações de conflito e mudanças, deparando-se com novas responsabilidades. Durante esse período os jovens Tikuna costumam casar-se, iniciando assim uma nova fase, com o início da vida sexual ativa e a mudança do rapaz para a casa do sogro, em posição de submissão à autoridade do pai de sua futura esposa. Tal situação se estende até o nascimento do segundo filho, época em que o casamento é considerado estável e determina a saída do casal da casa dos sogros, tornando-se uma unidade familiar independente.

Os relatos dos eventos nessa faixa etária enfatizam duas situações que parecem complementares: a presença acentuada de usuários de bebida alcoólica e a grande instabilidade do casamento que ocasiona, por sua vez, violência intensa dentro da unidade familiar (ERTHAL RMC, 2001).

Por fim, Erthal RMC (2001) aponta que apesar da menor incidência de suicídios entre o segmento feminino, o perfil da mulher Tikúna parece estar passando por um processo de reformulação, o que pode ocasionar mudança de padrão do evento para esta população. 
Outra característica diz respeito ao dia de ocorrência das mortes. A maioria se deu durante o final de semana. Estudo sobre a mortalidade por suicídio em São Gabriel da Cachoeira, sugere uma provável relação entre o aumento no número de casos de suicídio nos dias de fim de semana devido o consumo excessivo de álcool (SOUZA MPL e ORELLANA JDY, 2012). O mesmo texto pontua que as maiores taxas aparecem entre indívíduos solteiros. Tal inferência corrobora a literatura sobre suicídio, que lista o casamento/ estar em um relacionamento estável, como sendo um fator preventivo para o suicídio.

Quanto ao local de ocorrência da morte, os achados apontam para algum cômodo da residência do suicida. As mortes ocorridas fora da residência têm como ponto em comum a escolha de um local próximo a casa do indivíduo. Estudo realizado por Souza MPL e Orellana JDY (2013), apontou que os indígenas que cometeram suicídio, frequentemente morrem em seus domicílios e não nos serviços de saúde. Uma possível causa para isso, teria a ver com o tipo de método preferencial utilizado por estes indivíduos, o enforcamento, que se caracteriza por ser um evento tipicamente domiciliar e com alto grau de letalidade.

Embora os estudos sobre a relação entre suicídio e transtornos mentais sejam numerosos em contextos não indígenas, ainda são exíguos os estudos que abordem doença mental e suicídio entre populações indígenas, tornando inviável uma discussão sólida sobre tal associação no contexto estudado. No trabalho de Sousa AD (2016), a autora descreve a dificuldade que os entrevistados tinham em atribuir aos seus parentes distúrbios mentais.

\section{Suicídio indígena: métodos utilizados}

Os resultados demonstraram que o método comumente utilizado para perpretar o suicídio é o enforcamento (SOUZA MPL e ORELLANA JYD, 2012). Os autores ressaltam que pelo fato do suicídio ser um fenômemo mais prevalente entre o público masculino, o meio mais usado por eles costuma ser mais agressivo e letal.

A pesquisa realizada por Sousa AD (2016), cujo objetivo era analisar os significados atribuídos por indígenas, aos suicídios ocorridos na área urbana de São Gabriel da Cachoeira, mostrou que em todos os casos descritos o método utilizado para perpetrar o suicídio foi o enforcamento, tendo como instrumento principal a corda.

Alguns estudos apresentam o envenenamento como método alternativo para cometimento do suicídio. Os principais adeptos desse tipo de autoextermínio são os Sorowaha. De acordo com Poz JD (2000), os Sorowaha acreditam na existência de uma outra vida, após a morte. O caminho ideal de passagem para esta outra vida só seria alcançada após a ingestão do kunaha, veneno também conhecido como timbó (usado comumente na pescaria). Esta outra vida, seria um ótimo lugar para se viver, com muita alegria, onde ocorre o reencontro com os antepassados e onde ninguém envelhece. Entretanto, só chegariam a essa vida, aqueles que morrem por ingestão do timbó. Quem morre em decorrência da velhice é privado deste lugar e terá sua alma vagando sem destino. A velhice é tida como a segunda alternativa de vida, mais penosa ao verdadeiro caminho.

Outro método relatado para o cometimento do suicídio é o "feitiço". Pimentel SK (2006) descreve que os Guarani/ Kaiowá costumam levar em consideração a possibilidade de algum ataque de ordem sobrenatural atuar como desencadeador do ato suicida, levantando a possibilidade de que o indivíduo esteja sendo vítima de feitiço, tanto de algum inimigo (vivo, habitante deste mundo), quanto de assombrações (a alma de alguém que já morreu, ou um ser do mundo espiritual).

Pereira MM (2013), ouvindo indígenas do município de São Gabriel da Cachoeira, descreveu o "estrago" como sendo alguma coisa equivalente ao que os não indígenas chamam de "mal olhado" ou "quebranto", sendo representado por um desejo de malefício sobre a vida do indivíduo, sendo geralmente motivado por inveja.

Sousa AD (2016), também apresentou como fatores desencadeantes associados ao suicídio a "inveja" e o "sopro" ou "estrago". De acordo com Carvalho M (2011), o "sopro" tem como objetivo causar algum malefício à pessoa a qual ele foi destinado, já que o "sopro ou estrago é a manipulação de elemento natural para ingerência no mundo sobrenatural". 
Souza MLP e Ferreira LO (2014), abordaram essa temática, quando apontaram as dificuldades encontradas em se transpor o modelo biomédico vigente na sociedade ocidental sobre o suicídio, para o universo sociocultural particular das populações indígenas, sobretudo aquelas que vivem na região do Alto Rio Negro. Tendo como base uma revisão sobre essa questão, o estudo demonstrou que no sistema sociocósmico das populações indígenas, o suicídio é encarado como resultante da ação de um outro indivíduo, e não somente da pessoa que cometeu o ato.

No trabalho de Erthal RMC (2001), o suicídio é atribuído ao ato do "enfeitiçamento", podendo atingir os mais jovens, que são considerados os mais vulneráveis, mas também aqueles que que conseguiram acumular alguns bens que a maioria da comunidade da qual fazem parte, causando "inveja" àqueles que não usufruem da mesma vida.

Uma outra característica apontada pela autora, diz respeito ao suposto aumento da presença de álcool nas comunidades Tikuna que apresentaram mais casos de suicídio. Entretanto a autora deixa claro que é importante salientar que o consumo de bebidas alcoólicas deve ser avaliado sob a ótica dos significados diversos que esse uso pode assumir em sociedades indígenas (Erthal RMC, 2001).

Em uma outra situação, também descrita por Erthal RMC (2001), o suicídio ocorreria devido uma mudança abrupta no comportamento dos rapazes que até então apresentavam bom comportamento e rendimento na escola, após sofrerem uma reprimenda dos pais, geralmente associada ao consumo excessivo de bebida. Esse comportamento apresentado pelos jovens seria gerador de vergonha para os membros da família. Esses jovens costumam sentir muita raiva após terem sua atenção chamada pelos pais e passam a se comportar de uma forma considerada fora do normal, "perdendo a razão", tal fato, parece estar ligado ao consumo de grande quantidade de bebidas alcóolicas.

Assim, os resultados apresentados demonstram que nem sempre o indivíduo que tira a própria vida é reconhecido como o autor de sua morte, já que na cosmologia das populações indígenas, o indivíduo não é visto como parte isolada dos demais membros de sua família, mas se constitui como sujeito a partir das relações estabelecidas com eles e com os demais seres ou criaturas que vivem no cosmos (SOUZA MLP e FERREIRA LO, 2014; SOUSA AD, 2016).

\section{Suicídio indígena e o pós-morte: o que acontece com quem se mata?}

Diversos estudos antropológicos descrevem a visão de que vários povos indígenas não consideram a morte como o fim da vida e do indivíduo em questão. Assim, a morte decorrente do suicídio "também poderia remeter à transformação do status da pessoa indígena no contexto sociocósmico do qual ela faz parte" (SOUZA MPL e ONETY JÚNIOR RTS, 2014).

Os achados nos permitiram inferir que, dependendo do contexto étnico, a vida após a morte pode ser concebida como algo bom e desejável, como é o caso dos Sorowaha. Conforme citado anteriormente, Poz JD (2000) descreve que entre os Sorowaha, a existência nesse mundo é concebida como um estágio que antecede aquilo que eles consideram ser a verdadeira vida. Além disso, para os Sorowaha, a vida que se terá após a morte no mundo em que vivemos, dependerá totalmente da forma pela qual a pessoa morreu.

Já em diferentes grupos éticos, a morte através do suicídio é retratada de forma penosa, cheia de angústias e sofrimento. Povos indígenas da região do Alto Rio Negro acreditam que as pessoas que morrem em decorrência de suicídio não viveriam uma vida plena no "outro mundo", e sim sofreriam uma transmutação, podendo "retornar" na forma de animais, como paca, cutia, sapo, cobra, vivendo uma "sobrevida" a base de abacaxi, macaxeira e outros alimentos cultivados nas roças indígenas (SOUSA AD, 2016).

Estudo realizado em São Gabriel da Cachoeira no ano de 2016, cujo objetivo era analisar como os indígenas, devido as suas peculiaridades sociais e culturais significam as mortes decorrentes de suicídio, também trouxe evidências de que a preocupação com o pós-morte é uma constante entre estas populações. A autora relata que após o término "formal" da entrevista, uma das entrevistadas lhe confidenciou que sua maior preocupação era a de saber como o filho estaria "agora" (após a morte) (SOUSA AD, 2016). 
Tais fatos, ainda segundo Sousa AD (2016), evidenciam a aflição por parte dos familiares. Esse achado em particular aponta para as especificidades encontradas na significação do suicídio neste contexto, que de certa forma ao preocupar os familiares sobreviventes, geram-Ihes sofrimento e dor.

Souza MLP e Ferreira LO (2014), tendo como base estudos antropológicos anteriores, ressaltam a visão de que vários povos indígenas não consideram a morte como o fim da vida e do indivíduo em questão. Assim, a morte decorrente do suicídio "também poderia remeter à transformação do status da pessoa indígena no contexto sociocósmico do qual ela faz parte".

É importante salientar que essas hipóteses necessitam de estudos posteriores para sua verificação, mas também apontam para as dificuldades encontradas na significação do suicídio, no que diz respeito ao que ocorreria com o indivíduo quando ele comete tal ato em contextos culturalmente diferenciados (SOUZA MPL e ONETY JÚNIOR RTS, 2014).

\section{Suicídio indígena: uma problemática de saúde pública oculta.}

Ainda que o suicídio seja reconhecidamente um problema de saúde no Brasil e no mundo, as estatísticas sobre a ocorrência de suicídio entre povos indígenas ainda são escassas. Apenas algumas etnias apresentam a descrição do fenômeno em profundidade, envolvendo todas as suas particularidades, citam-se aí os trabalhos realizados com os Guarani, os Tikuna, os Sorowaha e algumas etnias da região do Alto Rio Negro (ORELLANA JDY, et al., 2013).

Os resultados apontaram para o que seria uma dificuldade em explicar/significar o suicídio a partir do ponto de vista do indígena. Ressaltando as dificuldades encontradas para se transpor o conceito biomédico de suicídio para o universo sociocultural particular das populações indígenas (SOUZA MLP e FERREIRA LO, 2014).

Tais achados destacam ainda a importância de se conhecer os diversos significados que constituem o fenômeno do suicídio, particularmente, no contexto indígena, como forma de contribuir para o enfrentamento de problemas de saúde pública vivenciados por essas populações.

Alguns trabalhos pontuam que outra possível explicação para o processo de visibilização da morte voluntária entre populações indígenas seria a baixa "representatividade" que os povos indígenas possuem no cenário nacional, uma vez que representam apenas $0,4 \%$ da população total do país (SOUZA MPL e ONETY JÚNIOR RTS, 2014).

O mesmo texto complementa essa questão com uma problemática ainda mais preocupante, quando relaciona a possibilidade da existência de casos subnotificados de suicídio, sobretudo no contexto indígena, por possuírem acesso dificultado aos serviços de saúde/ a maioria das mortes causadas por suicídio ocorrem nos domicílios, o que prejudica a valiação médica do evento e posterior notificação do óbito (SOUZA MPL e ONETY JÚNIOR RTS, 2014).

Por fim, estudos classificam o suicídio indígena não somente como um grave problema de saúde pública, mas um elemento que faz parte de todo um contexto sóciosanitário peculiar existente em populações especialmente vulneráveis, como é o caso dos indígenas, necessitando de de uma maior atenção por parte das autoridades nacionais e locais para combate/controle da situação (ORELLANA JDY, et al., 2013).

\section{CONSIDERAÇÕES FINAIS}

Faz-se necessário que se considere as especificidades socioculturais existentes nas populações indígenas no que diz respeito aos estudos sobre suicídio, sobretudo na formulação de estratégias de prevenção e assistência em saúde mental tanto para os suicidas em potencial, quanto para os sobreviventes. 


\section{REFERÊNCIAS}

1. BOTEGA NJ (coord). Prevenção do suicídio: manual dirigido profissionais da saúde da Atenção Básica. Ministério da Saúde; Organização Pan-americana da Saúde; UNICAMP; São Paulo, 2009.

2. BRASIL. Ministério da Saúde. Secretaria de Vigilância em Saúde - Suicidio. Saber, agir e previnir. Boletim Epidemiológico: Perfil epidemiológico das tentativas e óbitos por suicídio no Brasil e a rede de atenção à saúde. Brasil, 2017, vol 48, n30.

3. CARVALHO M. Inverso: diálogos indígenas no Alto Rio Negro. Pernambuco: Gráfica Facform: Instituto Antropos, $2011 ; 178 p$.

4. COUTINHO W. Suicídio indígena alto-rionegrino: circunstâncias e enigmas da morte voluntária no noroeste da Amazônia (Relatório Técnico) MP/AM, Manaus, 2011; 78p.

5. ERTHAL RMC. O suicídio Tikúna no Alto Solimões: uma expressão de conflitos. Cad. Saúde Pública, 2001; 17(2):299311.

6. FONSECA RMP. Revisão integrativa da pesquisa em enfermagem em centro cirúrgico no Brasil: trinta anos após o SAEP. Dissertação (Mestrado em Enfermagem) - Escola de Enfermagem da Universidade de São Paulo, São Paulo, 2008; 135p.

7. GANONG LH. Integrative Reviews of Nursing. Rev Nurs Health, 1987; 10(1): 1-11.

8. GRUBITS S, et al. Suicídios de jovens Guarani/Kaiowá de Mato Grosso do Sul, Brasil. Psicol. cienc. prof., 2011; 31(3): 504-517.

9. ORELLANA JDY, et al. Mortalidade por Suicídio: um enfoque em municípios com alta proporção de população autodeclarada indígena no Estado do Amazonas, Brasil. Rev. bras. epidemiol., 2013; 16(3): 658-669.

10. ORGANIZAÇÃO MUNDIAL DA SAÚDE. Prevención del suicidio: un imperativo global. Washington, DC: OPS, 2014.

11. ORGANIZAÇÃO PAN-AMERICANA DA SAÚDE. Folha informativa - Suicídio. 2018.

12. PEREIRA MM. Representações sociais sobre suicídio indígena em São Gabriel da Cachoeira-AM: um estudo exploratório. Dissertação (Mestrado multidisciplinar em saúde, sociedade e endemias na Amazônia) - Fundação Oswaldo Cruz/Instituto Leônidas e Maria Deane. Universidade Federal do Amazonas, Manaus, 2013; 127p.

13. PIMENTEL SK. Sansões e Guaxos: suicídio Guarani e Kaiowá: uma proposta de síntese. 2006. Dissertação (Mestrado em Antropologia Social) -Faculdade de Filosofia, Letras e Ciências Humanas. Universidade de São Paulo, São Paulo, 2006; 168p.

14. POZ JD. Crônica de uma morte anunciada: do suicídio entre os Sorowaha. Revista de Antropologia, 2000; 43(1): 89144.

15. REICHENHEIM ME, et al. Violence and injuries in Brazil: the effect, progress made, and challenges ahead. Lancet, 2011; 377(9781): 1962-75.

16. SOUSA AD. Suicídio na área urbana de São Gabriel da Cachoeira: estudo com autópsias psicossociais. Dissertação (Mestrado em Saúde, Sociedade e Endemias na Amazônia) - Universidade Federal do Amazonas, Manaus, 2016; 125P.

17. SOUZA MLP, FERREIRA LO. Jurupari se suicidou?: notas para investigação do suicídio no contexto indígena. Saúde e Sociedade, 2014; 23(3): 1064-1076.

18. SOUZA MLP, ONETY-JÚNIOR RTS. Caracterização da mortalidade por suicídio entre indígenas e não indígenas em Roraima, Brasil, 2009-2013. Epidemiol. Serv. Saúde, 2017; 26(4): 887-893.

19. SOUZA MLP, ORELLANA JDY. Suicide mortality in São Gabriel da Cachoeira, a predominantly indigenous Brazilian municipality. Revista Brasileira de Psiquiatria, 2012; 34(1): 34-37.

20. SOUZA MLP, ORELLANA JDY. Desigualdades na mortalidade por suicídio entre indígenas e não indígenas no estado do Amazonas, Brasil. J. bras. Psiquiatr, 2013; 62(4): 245-252.

21. SOUZA MT, et al. Revisão integrativa: o que é e como fazer. Einstein, 2010; 8(1): 102-106

22. WAISELFISZ JJ. Mapa da Violência no Brasil 2011: Os jovens do Brasil. Instituto Sangari, São Paulo, 2011. 